\title{
EVOLUTION OF MASSIVE STARS FROM HELIUM BURNING TO THE START OF NEUTRONIZATION
}

\author{
(Abstract)
}

W. DAVID ARNETT

Dept. of Astronomy, University of Texas, Austin, Tex., U.S.A.

Evolutionary sequences have been constructed beginning at helium burning and ending in the hydrodynamic collapse of the core when neutronization begins. Details of these models during silicon burning as well as at the onset of neutronization are presented. The connection of these results with the calculations of Wilson (1971) and Arnett (1967) is discussed. Calculations by Ostriker and Gunn (1971) and Falk and Arnett (1972) provide the beginning of a detailed understanding of supernova light curves. Taken together a coherent and continuous picture of stellar evolution through supernova explosion and pulsar formation can be obtained. While the theory still needs much more work, the gaps so obvious a few years ago are disappearing.

\section{References}

Arnett, W. D.: 1967, Can. J. Phys. 45, 1621.

Falk, S. W. and Arnett, W. D.: 1973, Astrophys. J. 180, L65.

Ostriker, J. P. and Gunn, J. E.: 1971, Astrophys. J. 164, L95.

Wilson, J. R.: 1971, Astrophys. J. 163, 209. 